From 'what's wrong with you?' to 'what's happened to you?':

An introduction to the Special issue on the Power Threat Meaning framework

\author{
David J. Harper \\ Mental Health \& Social Change Research Group \\ School of Psychology \\ University of East London \\ Water Lane \\ London E15 4LZ \\ $\boldsymbol{\&}$ \\ John Cromby \\ ULSB \\ University of Leicester \\ University road \\ Leicester LE1 7RH
}

\title{
Keywords:
}

Power Threat Meaning Framework; Diagnosis; DSM-5; Medicalization 
From 'what's wrong with you?' to 'what's happened to you?':

An introduction to the Special issue on the Power Threat Meaning framework

\begin{abstract}
The Power Threat Meaning Framework (PTMF), published by the British Psychological Society (BPS) in 2018, is an attempt to address the question of how we might understand what Harry Stack Sullivan called 'problems in living' other than by using psychiatric diagnostic systems. How might we best conceptualize emotional distress and behaviour which might concern or trouble others? We describe the context within which the PTMF was developed and explain some of its key elements before giving an overview of the articles in this special issue.
\end{abstract}




\section{From 'what's wrong with you?' to 'what's happened to you?': An introduction to the Special issue on the Power Threat Meaning framework}

The Power Threat Meaning Framework (PTMF), published by the British Psychological Society (BPS) in 2018, is an attempt to address the question of how we might understand what Harry Stack Sullivan called 'problems in living' other than by using psychiatric diagnostic systems. How might we best conceptualize emotional distress and behaviour which might concern or trouble others? It was the result of five years of work, funded by the BPS Division of Clinical Psychology, by an author team including psychologists and psychiatric survivor campaigners. The work to produce the framework began in early 2013, months before the publication, in May that year, of the American Psychiatric Association's (APA) DSM-5 (American Psychiatric Association, 2013). This special issue includes a series of articles on the PTMF aimed at elaborating some of its central ideas. Before giving an overview of the articles we first describe the context within which the PTMF was developed and explain some of its key elements.

There is widespread dissatisfaction with psychiatric diagnostic systems like DSM-5 and the World Health Organisation's (2018) ICD-11, and the criticisms are well-known so we will not repeat them here (for a comprehensive summary see Johnstone \& Boyle 2018a, chapter 1). What appeared to be new in 2013 was a wave of widespread public discussion about diagnosis. Days before the publication of DSM-5 there was a remarkable public debate between Dr Thomas Insel, Director of the National Institute for Mental Health (NIMH) and Dr David Kupfer who chaired the APA taskforce producing DSM-5. Insel stated in a blog that the NIMH would be re-orienting its research away from the DSM's diagnostic categories 
as they lacked scientific validity. Four days later, the APA published a press release from Kupfer noting that psychiatry had so far failed to find the much-vaunted biomarkers that Insel (along with most of psychiatry) was advocating. This debate reached the pages of the New York Times which noted that the publication of DSM-5 had been met with 'unprecedented questioning'. Indeed, DSM-5 noted that the APA feedback website received over 13,000 comments and thousands of organized petitions (American Psychiatric Association, 2013).

In North America many practitioners are in private practice and those who adopt a nonpsychiatric therapeutic approach report experiencing dilemmas since they are required to give a DSM diagnosis in order to be reimbursed (Strong, Gaete, Sametband, French \& Eeson, 2012). In contrast, in the UK, the vast majority of clinical psychologists work in the National Health Service (NHS), which also funds their training. As a result, they are not dependent on reimbursement as the NHS is free to access. Although British psychiatrists use ICD-11 categories regularly in their work, in the UK formulation is a much more central organizing construct for practicing psychologists (Johnstone, 2013). Moreover, there is a strong tradition in British psychology which is critical both of psychiatric diagnosis and of medicalization. This was evident when the BPS submitted responses during the DSM-5 consultation process which were openly critical of key assumptions underpinning psychiatric frameworks (Lane, 2012). Although not all British psychologists agree with such critiques (see for example the letters pages of the BPS in-house magazine The Psychologist (https://thepsychologist.bps.org.uk/) between June-October 2013), many do.

It was in this context that the author team set to its work, eventually producing two substantial reports: a 411 page 'main' document synthesizing research findings from a 
number of key domains (Johnstone \& Boyle 2018a); and a 136 page 'overview' version aimed at practitioners (Johnstone \& Boyle 2018b). ). The main document summarizes critiques of diagnosis, identifies the PTMF's philosophical and theoretical commitments, describes the central role of meaning and narrative, summarizes evidence regarding both social influence and biology (and sets out ways of integrating both sets of evidence), and describes how the PTMF can be used to identify patterns in distress and distressing behaviors. Both are freely available via the BPS PTMF website:

https://www.bps.org.uk/power-threat-meaning-framework

The central ideas of the PTMF are that adversities in life are patterned by social inequality and, thus by the negative operations of power in the lives of individuals. These adversities pose threats, and we react to those threats with embodied threat responses. Within a medical or diagnostic framework these threat responses get decontextualized and viewed as symptoms of underlying disorders. In contrast, the PTMF proposes that they are meaningful and potentially intelligible responses to complex combinations of toxic circumstances and events. However, the links between threats and responses are not linear and predictable, for a number of reasons. Firstly, they are dynamically mediated by personal meanings and agency, and a threat response may serve different functions for a person at different times and in different contexts. Secondly, mixtures of exacerbating and ameliorating factors also complicate the picture. Lastly, they are contingently and synergistically related to other threats and responses. As a result, the PTMF suggests we move from the kind of medicalized disorderinformed or diagnosis-based approach, which is organized by the question 'what is wrong with you?', to one organized by questions such as 'what happened to you?', 'how did it affect you?' and 'what did you find yourself doing to survive?' 
Whilst the PTMF provides a novel and coherent way of conceptualising both distress and behaviours that are distressing to others, it is not a new model for therapy and nor is it specifically aligned with any one therapeutic perspective or approach. It has implications for professional psychological work across the board but does not proscribe in advance how practitioners should draw upon it. As the main document notes:

"the framework is not tied to a particular level of explanation (social, psychological or biological) or to a specific theoretical orientation such as cognitive, behavioural or systemic. Thus, while we suggest it can be used as it stands, it can also be seen as a meta-framework within which many existing models and bodies of evidence can be accommodated" (Johnstone \& Boyle 2018a, p.5)

In addition to these implications for therapeutic practice, the PTMF suggests constructive alternatives with regard to service design and commissioning, professional training, research, service user involvement, peer support, public information, and social and public policy.

\section{The Articles}

There are seven articles in this special issue, six of which are written by PTMF co-authors. However, the first article is written by members of the SHIFT (Self Help Inspiring Forward Thinking) recovery community based in Portsmouth, England. SHIFT is a peer-led group of people with lived experience of mental and emotional distress. They describe how, after encountering the PTMF, they decided to work through the overview document together, 
seeking to understand the ideas in it and apply it to their own lives. We found it an inspiring read and its message of the need to make these ideas much more accessible is well-taken. It has been gratifying to hear about how people with lived experience have made use of the framework, and this article by SHIFT complements work such as that undertaken by the Mental Health Peer Support Group in York, England (Griffiths, 2019).

The second, third and fourth articles elaborate and clarify central aspects of the PTMF. The second article, by Lucy Johnstone, one of the two lead authors of the PTMF, discusses the patterns of distress that the PTMF offers in place of diagnostic categories. Johnstone explains how these patterns are fundamentally different from psychiatric diagnoses. Because they are organised by meaning, not (presumed and still hypothetical flaws in) biology, these patterns are necessarily embedded in history and include elements of culture. And because they describe things that people find themselves doing or experiencing (in response to the meanings of threats caused by imbalances or abuses of power), they avoid either positioning those in distress as passive victims of biological misfortune, or possessing them as wholly culpable (by virtue of their actions or omissions) for their own misery.

The third article, by Mary Boyle, the other lead author of the PTMF focuses on how power is conceptualized in the framework. She describes the different types of power it discusses (e.g. economic power, interpersonal power etc.). She argues that power not only affects the likelihood of experiencing adversity but also the kinds of threat responses we have available to us and, how ideological power can influence the kinds of ideas which are culturally available to us to understand distress and troubling conduct. 
The fourth article, by PTMF contributing author John Cromby, examines another key concept in the framework: meaning. Cromby situates the PTMF's concept of meaning with respect to others discussed in this journal. He explains how, for the PTMF, meaning is the complex and continuously emerging product of a diverse set of constituent elements that typically include language, feeling, habit, materiality and culture. Since none of these elements are wholly controlled or possessed by any one individual, the personal meanings we encounter in everyday life are never straightforwardly a matter of our own personal choice. Instead, for the PTMF meaning is something that, in the late John Shotter's words, we both 'make and find'.

In the fifth article, PTMF contributing authors John Read and David Harper argue that the increasing amount of research pointing to links between adversity and distress, supports the PTMF approach. They observe that, whilst psychiatric diagnostic categories appear to provide an explanation for distress, in practice they do not, as they often obscure these links. A central assumption of the 'mental illness is an illness like any other' approach to combating the stigma and prejudice faced by psychiatric service users is that a medical understanding of distress will reduce stigma. However, as Read and Harper show, the empirical evidence points in the opposite direction: the medical model is associated with more, not less, stigma. Finally, they suggest some ways in which mental health services could be transformed in line with the PTMF and other innovations like the increasingly popular 'Trauma-informed Care' approach. 
In the sixth article, David Harper discusses the different ways in which the narrative construct is used in the PTMF to refer to personal narratives, cultural narratives and as a metatheoretical language to synthesize insights from the 14 different theoretical perspectives which influenced its development. After discussing ways in which these uses may differ from therapeutic approaches likely to be familiar to readers of this journal, Harper then moves on to examine why a medical narrative is so dominant in the public conversation about mental health, drawing on concepts from media studies, political science and feminist philosophy. He concludes by arguing that we need to re-balance the public conversation and makes some suggestions for how therapists as well as a range of other groups can support such a process.

In the final article, PTMF contributing author David Pilgrim explores the relevance for the PTMF of critical realist philosophy, drawing out some of the ways in which the Framework is broadly compatible with its precepts. In particular, Pilgrim considers the flaws of both positivism and relativistic versions of constructionism/constructivism. Rejecting both of these, he locates the PTMF in a moral context where facts and values cannot straightforwardly be easily separated, a context wherein psychiatry (and sometimes psychology) frequently functions to legitimate coercive social control.

\section{Reception and Uptake}

The PTMF has had a significant impact on the mental health field since its publication in January 2018. Already it has been cited over 100 times according to Google Scholar. There has been debate and, inevitably, some criticism - see Johstone et al (2019) for a summary and reflection. Members of the author team have delivered talks and training workshops across 
the UK and in countries including Denmark, Spain, Ireland, Greece, New Zealand and Australia. The Overview version has been translated into Spanish as El Marco de Poder, Amenaza y Significado: Publicación abreviada (http://aen.es/wpcontent/uploads/2018/08/El-Marco-de-Poder-Amenaza-y-Significado-1.pdf) and other translations are in progress. The January 2019 special issue of Clinical Psychology Forum included articles from the UK and the Republic of Ireland showing how it had been used in professional training (social workers, teachers and clinical psychologists), and in a range of applied or service settings (peer-support, youth work, prison, courses for women subjected to abuse, and an autism and learning disability team). The February 2019 special issue of Clinical Psychology Forum, on improving services for trauma-related dissociation, included two articles discussing how the PTMF was combined with a trauma-informed approach to develop new care pathways. At the January 2020 BPS Division of Clinical Psychology conference a symposium on the PTMF heard how it was a useful resource for the coproduction of clinical formulations with service users (Bostock \& Armstrong, 2020), how it was being used in work with women survivors of sexual violence and abuse (Watson, 2020) and how it was being used to support in-patient staff on psychiatric hospital wards to develop team formulations (Nikopaschos \& Burrell, 2020).

\section{References}

American Psychiatric Association (2013) Diagnostic and statistical manual of mental disorders, fifth edition (DSM-5), Washington, VA: American Psychiatric Publishing. 
Bostock, J., \& Armstrong, N. (2020, January 22). Using the Power Threat Meaning Framework to co-construct formulations and action. [Paper presentation]. Division of

Clinical Psychology Annual Conference 2020: New Adventures in Clinical Psychology:

Personal, Professional and Political Partnerships. Solihull, England.

https://www.bps.org.uk/events/division-clinical-psychology-annual-conference-2020

Griffiths, A. (2019, January). Reflections on using the Power Threat Meaning Framework in peer-led systems. Clinical Psychology Forum, 313, 9-14.

Johnstone, L. (2013). Diagnosis and formulation. In J. Cromby, D. Harper \& P. Reavey Psychology, Mental Health and Distress. Basingstoke: Palgrave Macmillan.

Johnstone, L. \& Boyle, M., with Cromby, J., Dillon, J., Harper, D., Kinderman, P., Longden, E., Pilgrim, D. \& Read, J. (2018a). The Power Threat Meaning Framework: Towards the identification of patterns in emotional distress, unusual experiences and troubled or troubling behaviour, as an alternative to functional psychiatric diagnosis. Leicester: British Psychological Society.

Johnstone, L. \& Boyle, M., with Cromby, J., Dillon, J., Harper, D., Kinderman, P., Longden, E., Pilgrim, D. \& Read, J. (2018b). The Power Threat Meaning Framework: Overview. Leicester: British Psychological Society. 
Johnstone, L., Boyle, M., Cromby, J., Dillon, J., Harper, D., Kinderman, P., . . Read, J. (2019). Reflections on responses to the Power Threat Meaning Framework one year on. Clinical Psychology Forum, 313, 47-54.

Nikopaschos, F., \& Burrell, G. (2020, January 22). Using the Power Threat Meaning Framework (PTMF) to inform team formulation in an adult acute inpatient setting. [Paper presentation]. Division of Clinical Psychology Annual Conference 2020: New Adventures in Clinical Psychology: Personal, Professional and Political Partnerships. Solihull, England. https://www.bps.org.uk/events/division-clinical-psychology-annual-conference-2020

Lane, C. (2012). Arguing over DSM-5: The British Psychological Society has serious concerns about the manual. Psychology Today, 20 June. Retrieved from: https://www.psychologytoday.com/gb/blog/side-effects/201206/arguing-over-dsm-5-thebritish-psychological-society-has-serious-concerns

Watson, G. (2020, January 22). Naming power and power abuses: using the PTMF to inform work with women survivors of sexual violence and abuse. [Paper presentation]. Division of Clinical Psychology Annual Conference 2020: New Adventures in Clinical Psychology: Personal, Professional and Political Partnerships. Solihull, England. https://www.bps.org.uk/events/division-clinical-psychology-annual-conference-2020 
World Health Organisation (2018) International classification of disease, Eleventh revision.

Geneva, World Health Organisation. 